\title{
Paty Criterion
}

National Cancer Institute

\section{Source}

National Cancer Institute. Paty Criterion. NCI Thesaurus. Code C67068.

Criteria established for assigning high-frequency microsatellite instability (MSI-H) in cancers requiring that at least two of three markers (BAT25, BAT 26, D2S123) score positive for MSI. 